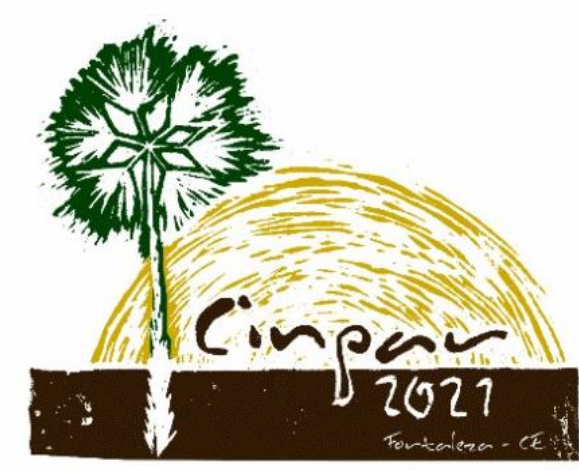

XVII Congresso Internacional sobre Patologia e Reabilitação das Construções

XVII Congreso Internacional sobre Patología y Rehabilitación de las Construcciones

XVII International Conference on Pathology and Constructions Rehabilitation

FORTALEZA (Brasil), 3 a 5 de junho de 2021

https://doi.org/10.4322/CINPAR.2021.108

\title{
Investigação Sobre a Origem de Erros e Inconformidades em Obras de Engenharia Civil
}

\author{
Rubia Nunes VIEIRA ${ }^{1}$, Gláucia Nolasco de Almeida MELLO² \\ ${ }^{1}$ Pontifícia Universidade Católica de Minas Gerais, Belo Horizonte, Brasil, rubia.nunes@sga.pucminas.br \\ ${ }^{2}$ Pontifícia Universidade Católica de Minas Gerais, Belo Horizonte, Brasil, gnamello@pucminas.br
}

Resumo: O presente artigo propôs a realização de um estudo de obras de engenharia civil que apresentaram falhas e não conformidades. Buscou-se identificar em qual das três fases originam-se os erros: concepção e projeto, execução e manutenção. A fase da execução engloba desde a aplicação de materiais inadequados e até mesmo a verificação do processo executivo adequado para o modelo. Discutiu-se e abordou-se a sistemática de quantificar em qual ou quais dessas três etapas há maior ocorrência e/ou incidência dos erros construtivos. A metodologia utilizada foi à revisão sistemática da bibliografia visando sustentar o objeto desse estudo por meio das contribuições científicas de diversos autores e publicações sobre o tema. Nesse sentido, aplicou-se como metodologia científica a busca por artigos de investigação e estudos de casos, disponíveis na plataforma de periódicos CAPES e em revistas brasileiras, renomadas e reconhecidas pelo conteúdo de obras da Engenharia Civil. Trabalhou-se com a expectativa de apontar e identificar a origem dos erros e inadequações no processo construtivo, dentro de um vasto universo de obras. Assim, o crivo aplicado para a seleção de referências se deu inicialmente a partir dos títulos encontrados e, posteriormente, pela realização de novo filtro pela leitura do resumo. E, por último, por meio de uma leitura integral. Foram selecionados, inicialmente, 654 artigos, e deste conjunto foram escolhidos 113 para compor o banco de informações da revisão bibliográfica final. Assim, constatou-se que as origens do problema construtivo residem predominantemente na fase de execução da obra, com maiores registros de eventos que apontavam inconformidades e discordâncias construtivas, seguindo-se as fases projetual e manutenção, nessa ordem, apresentando números bastante significativos. Observou-se ainda que as manifestações de erros e não conformidades construtivas estão presentes em mais de uma etapa, com a significativa porcentagem de $59 \%$ dos acontecimentos da amostra desta investigação.

Palavras-chave: Engenharia de estruturas, projeto e execução de obras, fases do processo construtivo, erros e inconformidades.

Abstract: This research proposed a study of civil engineering works that presented flaws and non-
conformities. It was tried to identify in which of the three phases the errors originate: conception and
design, construction, and maintenance. It was discussed in which of three phases there is a greater
occurrence and/or incidence of constructive errors. The methodology used was bibliography's systematic
review to support the object of this study through the scientific contributions of several authors and
publications on the subject. In this sense, the search for articles and case studies, available on the CAPES
platform journals and in some Brazilian journals was applied as a scientific methodology. Research was
carried out with the expectation of pointing out and identifying the source of errors and non-conformities
in the construction process, within a vast universe of works. Thus, selection criteria applied for choosing
references was initially based on the article's titles and, later, through the realization of a new filter by
reading the summary. And, finally, through a full reading. Initially, 654 articles were selected, and from this
set, 113 were chosen to compose the database of the final bibliographic review. Thus, it was found that the
origins of the construction problem lie predominantly in the construction phase, with greater records of 
events, followed by the design and maintenance phases, in that order, presenting quite significant numbers. It was also observed that manifestations of errors are present in more than one phases, with a significant percentage $(59 \%)$ of the events in the sample of this investigation.

Keywords: Structural engineering, design and construction of works, construction process phases, errors and non-conformities.

\section{Introdução}

O mercado da construção civil é um campo de acentuada importância no aspecto social e econômico, pois, tem o papel de produzir obras destinadas à infraestrutura, moradia, atividades comerciais, industriais etc., cooperando para a geração de emprego e renda, abrindo caminhos para o progresso social.

Para Isoppo e Bernardino (2017), tais demandas fez com que a engenharia civil passasse por um processo de alteração e evolução desde sua origem até os tempos atuais. Dessa forma, foi exigido que todos os atores envolvidos se readequassem, operacional e estrategicamente, dos processos construtivos (VANNI, GOMES e ANDERY, 2016). Nesse sentido, procurar-se-ia, portanto, minimizar a frequência de falhas construtivas, além de diminuir perdas e reduzir tempos ociosos, promovendo o aumento da produtividade (KATO, 2002).

Contudo, percebe-se, ainda hoje, a ocorrência significativa de erros em projetos estruturais, a baixa capacitação da mão de obra atuante na fase de execução, a ocorrência de falhas na gestão de obras, o desleixo com a manutenção das estruturas, dentre outros problemas relacionados ao processo de construção civil.

Dentro deste contexto, o presente artigo apresenta a avaliação de anormalidades construtivas em obras de engenharia civil, com o intuito de responder à seguinte questão: Em qual das fases do processo de construção civil há maior ocorrência de falhas? O principal objetivo da pesquisa é alertar e contribuir para o aprimoramento técnico e profissional buscando a melhoria da qualidade dos serviços de engenharia, seja na fase de projeto, construção ou manutenção.

\section{Referencial Teórico}

Existem cinco etapas que compõem o processo construtivo, são elas: planejamento; elaboração de projetos; preparação para execução; execução e, por fim, a etapa de uso (FABRÍCIO, 2002; ROMANO 2003). A primeira fase do processo de projeto refere-se ao planejamento que compreende a concepção e todos os estudos e análises técnicas e econômicas preliminares, tendo como pré-requisito o planejamento estratégico da construtora e/ou incorporadora responsável pela obra; informações dos clientes, e por último, dados do mercado quanto ao produto a ser ofertado (TZORTZOPOULOS, 1999).

Na segunda fase no qual é tratada a elaboração do projeto, se define a solução mais adequada e viável para a construção do produto da construção civil, estabelecendo todas as especificações necessárias por meio de desenhos gráficos, memoriais descritivos, entre outros dados capazes de definir claramente todas as características do produto a ser construído, de modo a subsidiar a sua execução (ASBEA (2000) apud ROMANO, 2003). A terceira etapa, conforme Romano (2003) está relacionada à preparação para execução dos projetos elaborados. Assim, ocorre à transição entre o projeto e a execução, havendo interação entre fases de concepção, elaboração de projetos e preparação.

A quarta fase, trata da execução propriamente dita e destina-se ao estágio do processo produtivo, no qual há a realização de todas as atividades em prol da materialização do produto. Já a quinta e última fase compreende um grupo de atividades indispensáveis para manter o bom desempenho ao longo do tempo, englobando desde a limpeza e substituição ou restauração de elementos com baixo desempenho, até mesmo consertos de defeitos originados durante alguma das etapas construtivas, em prol do bom desempenho estrutural (CREMONINI, 1988; CBIC, 2013). 
Dentre as principais causas de incidentes em obras de engenharia, têm-se os erros de projeto e de execução, ocasionados por diversos fatores como imperícia, negligência ou imprudência. Contudo, há situações e incidentes motivados por condições adversas e fatores imponderáveis, como os ocorridos em catástrofes naturais (MARCELLI, 2007).

Quanto aos erros cometidos na fase projetual, ocorrem no momento da concepção da obra, e segundo Vanni et al. (1999) e Correia et al. (2018), o segredo para o bom desempenho de uma obra está no projeto, considerado como carro-chefe para racionalização e onde deve-se avaliar particularidades do processo para que se obtenha sucesso na etapa de construção. Nesse sentido, para Correia et al. (2018), a fase de projeto é um eixo determinante no panorama de qualquer obra sendo necessário uma análise cautelosa, de forma conciliada e em harmonia com o setor de produção.

Com relação aos erros nos processos executivos, para Bomtempo (2017), os mesmos acontecem no decorrer, ou no desenvolvimento de uma obra e algumas falhas podem ocasionar danos à estrutura, levando-a ao colapso total ou parcial.

As manifestações patológicas ocasionadas na concepção de projetos são aquelas que resultam de um planejamento inadequado ou falhas técnicas, seja por falta de conhecimento ou descuido, podendo originar de uma falha nas especificações do anteprojeto ou até mesmo na elaboração do projeto executivo, o que torna imprescindível a importância de uma gestão eficaz na fase de execução das obras (TRINDADE, 2015). Os problemas patológicos construtivos também podem surgir devido à utilização incorreta na etapa do pós-obra, mesmo que se tenha atingido na etapa de execução, a ausência de anomalias, em decorrências de modificações na estrutura, podendo provocar a instabilidade da estrutura, ocasionar trincas, deformações, perda de resistência etc. (IANTAS, 2010; TRINDADE, 2015).

Conforme Oliveira (2013), algumas pesquisas apontam que a maior parte das manifestações patológicas surge durante a fase de execução da obra, oposto ao que ocorre na Europa, em que o maior percentual dos problemas se manifesta na fase projetual, assim como, abordado por Helene (2003), em que a fase de concepção de projetos é a etapa construtiva que ocorre erros em maior incidência, como ilustrado no Gráfico 1.

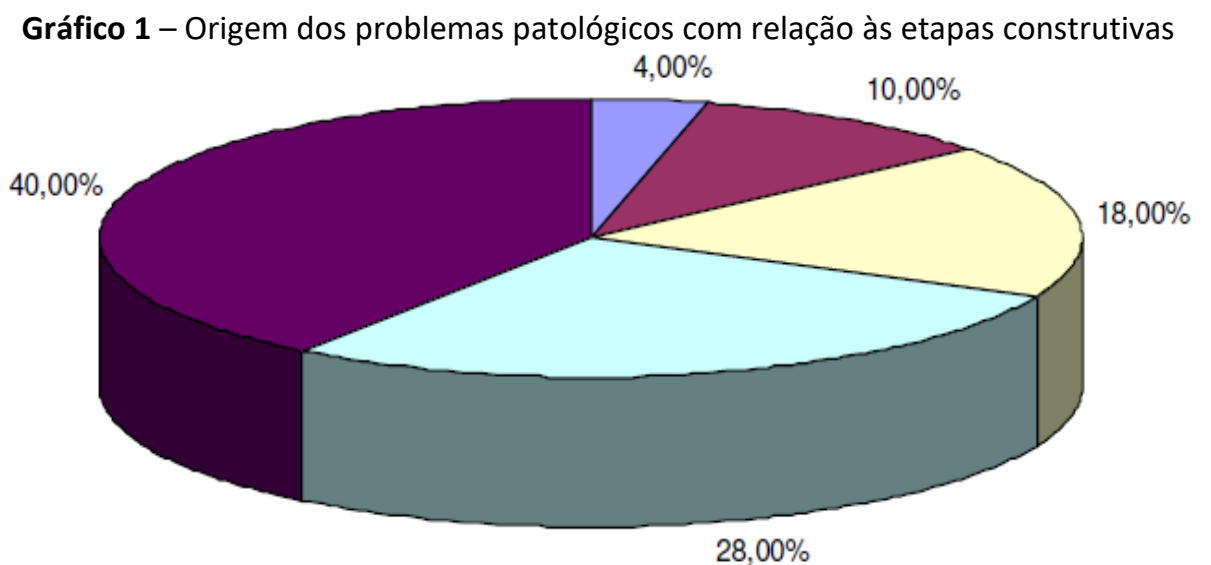

$28,00 \%$

$\square$ Planejamento $\square$ Uso $\square$ Materiais $\square$ Execução घ Projeto

Fonte: (HELENE, 2003, p. 25)

\section{Metodologia}

A metodologia adotada na pesquisa em questão é a revisão bibliográfica sistemática relacionada a obras de engenharia civil que apresentaram falhas, manifestações patológicas, erros ou não conformidades, para base de análise, apuração dos resultados e posteriormente conclusões a respeito do objeto de estudo desta investigação. 
Assim, foi realizada a busca por artigos e estudos de casos em revistas brasileiras e publicações em bases disponíveis na plataforma de periódicos CAPES reconhecidos pelo seu conteúdo e teor dentro no know hall de engenharia civil, que identificassem a origem das falhas, erros e inconformidades no processo construtivo dentro do universo de obras de engenharia.

Ressalta-se ainda que, não se aplicou quaisquer restrições quanto ao tipo de obra, especificação de estrutura, técnica ou metodologia construtiva, seja ela obra de arte, predial, obras de terra, estradas, infraestrutura, estruturas de aço, madeira, concreto, entre outras.

Nesse cenário, foram priorizados os resultados que referenciavam as origens do problema construtivo dentre suas três etapas (projeto, execução e manutenção), para que fosse possível atender a finalidade desse estudo.

Durante a leitura da bibliografia, observou-se os apontamentos de falhas advindas da fase de planejamento e projeto, do processo de execução e do período de manutenção. Então foi possível apurar e analisar em qual ou quais das etapas originam-se, com maior ocorrência, as não conformidades.

Selecionou-se, para compor esta pesquisa, cinco bases da plataforma de periódicos CAPES, sendo elas: American Society of Civil Engineers; Engineering Journals (ProQuest); IEEE Xplore; ScienceDirect (Elsevier) e Taylor \& Francis Online. Com relação às revistas brasileiras foram consultadas a Revista Concreto e Construções e a Revista de Materiais e Estruturas do IBRACON. Isto posto, selecionou-se ainda como termos de busca, expressões nos idiomas inglês e português.

Além disso, definiu-se ainda como período de pesquisa as publicações realizadas nos últimos cinco anos, ou seja, entre o intervalo dos anos de 2015 a 2020 com o propósito de trazer mais contemporaneidade à conjuntura da construção civil, pois, a origem das falhas e erros construtivos podem se submeter a mudanças frente a inserção de novas práticas, tecnologias etc.

Optou-se ainda por realizar a busca de dados e informações inicialmente pelo título das publicações, e, após esse crivo, fez-se uma nova triagem a partir da leitura do resumo dos artigos e estudos de casos, e, por último, realizou-se um filtro final de todo o material, selecionando-os por meio da leitura na integralidade.

A Tabela 1 apresenta um resumo dos resultados obtidos em cada fase da busca dessa investigação, desde a seleção por título, resumo e da triagem na fase de leitura.

Tabela 1 - Resumo dos resultados de busca de artigos científicos e estudos de casos (Metodologia)
\begin{tabular}{|c|c|c|c|c|}
\hline Base CAPES / Revista Brasileira & Idioma & Qtde Selecionados & Qtde Selecionados & Qíde Selecionados Título \\
por Resumo & por Leitura \\
\hline American Society of Civil Engineers & Inglês & 207 & 50 & 34 \\
\hline ScienceDirect (Elsevier) & Inglês & 51 & 12 & 11 \\
\hline Engineering Journals (ProQuest) & Inglês & 76 & 13 & 10 \\
\hline Taylor \& Francis online & Inglês & 238 & 43 & 31 \\
\hline IEEE Xplore & Inglês & 15 & 3 & 3 \\
\hline Revista Concreto e Construções & Inglês / Português & 53 & 31 & 23 \\
\hline Revista IBRACON de Estruturas e Materiais & Português & 14 & 6 & 1 \\
\hline Total: & & 654 & 158 & 113 \\
\hline
\end{tabular}

Fonte: Elaborado pela autora (2020).

Planilhas construídas com o auxilio do programa Microsoft Office Excel, foram utilizadas para organizar as informações, gerir os dados, realizar os levantamentos, além de, promover relatórios a partir de tabelas e gráficos capazes de apoiar as análises alusivas ao objeto dessa pesquisa (apêndices $A, B, C$ e D e Quadros 1 a 6, VIEIRA, 2020).

\section{Resultados e discussões dos resultados}

Essa pesquisa buscou identificar a origem dos erros e inconformidades existentes no processo construtivo, em que, priorizou-se apurar em qual das três etapas (projeto, execução e manutenção) predominam-se a ocorrência de falhas e não conformidades em obras de engenharia. 
Sob esse aspecto, é possível perceber pelo Gráfico 2 e Tabela 2 que a etapa onde originam-se predominantemente os erros, encontra-se na fase de execução onde há 75 ocorrências que correspondem a $38 \%$ dos eventos, isto é, mais de um terço dos apontamentos.

Em seguida, tem-se ocupando o segundo lugar a fase de projeto com $32 \%$ dos casos, percentual este muito próximo a terceira e última colocação representada pela etapa de manutenção que atingiu $30 \%$.

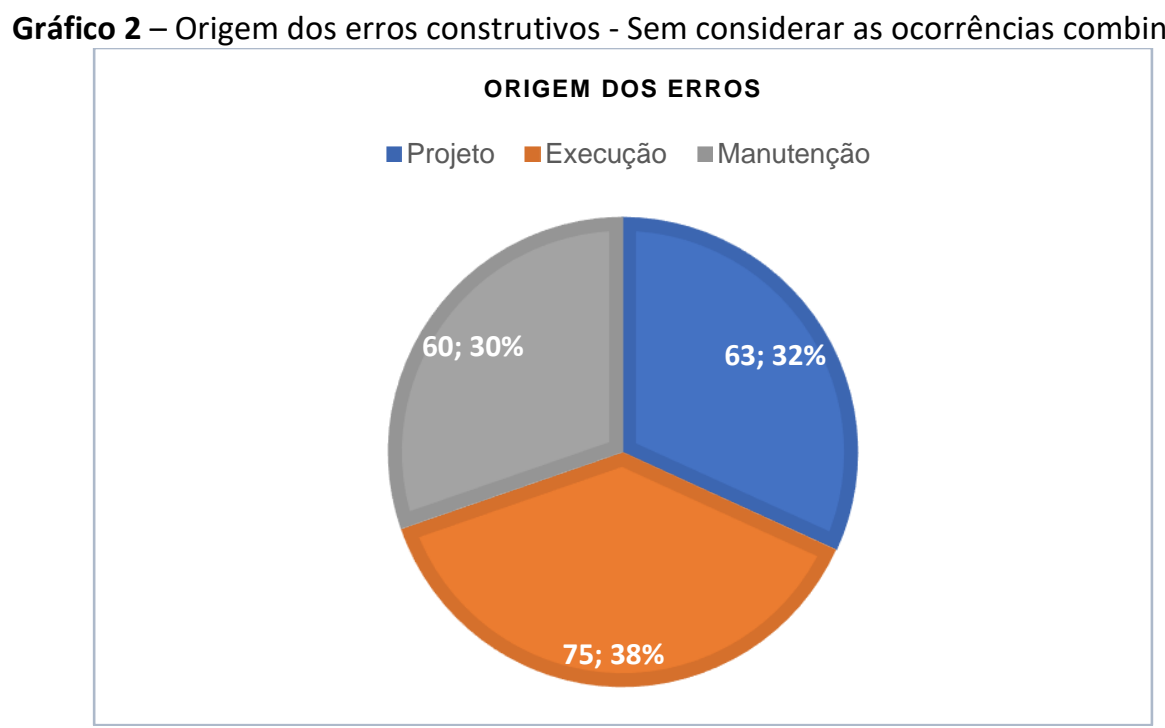

Fonte: Resultado da pesquisa (2020).

Tabela 2 - Origem dos erros construtivos - Sem ocorrências combinadas

\begin{tabular}{ccc} 
Origem & Quantidade de Apontamentos & Percentual (\%) \\
\hline Projeto & 63 & $31,8 \%$ \\
Execução & 75 & $37,9 \%$ \\
Manutenção & 60 & $30,3 \%$ \\
& 198 & $100,0 \%$ \\
\hline
\end{tabular}

Fonte: Resultado da pesquisa (2020).

Todavia, é possível observar ainda na Tabela 2 que há um total de 198 apontamentos, ou seja, um número superior ao quantitativo de artigos e estudos de casos selecionados para essa amostragem, assim, tal divergência é justificada por obras que apresentaram ocorrências de falhas combinadas em mais de uma etapa, isto é, ocorreram falhas em diversos períodos durante o processo construtivo.

Portanto, considerando esse novo contexto foi imprescindível aprofundar este estudo e analisar as situações onde ocorreram erros e falhas em apenas uma das etapas, bem como, as ocasiões que por sua vez encontram-se manifestações de discordâncias em mais de uma fase do processo construtivo.

Para tanto, foi possível perceber por meio do Gráfico 3 e da Tabela 3 que, quando os números são analisados de forma isolada, ou seja, quando agrupamos as obras que apresentaram erros construtivos fundamentados na(s) mesma(s) origem(gens), a etapa projetual, em um cenário em que ocorreu inadequações apenas nesse momento, aparece com 17 eventos, se comparada as demais ocorrências que exibiram erros em apenas uma das fases.

Com relação aos casos em que foram sinalizados a origem dos problemas construtivos na fase de manutenção apenas, obteve-se o segundo lugar com 16 apontamentos, seguindo da etapa de execução com apenas 13 indicações de inconformidades.

Assim, observa-se uma mudança de posicionamentos comparando-se os resultados do Gráfico 2 com o Gráfico 3, entretanto, cabe ressaltar ainda que, ambas as análises estão corretas, havendo apenas diretrizes e parâmetros díspares, onde, ora analisam-se os apontamentos das falhas de modo geral, onde, foi 
contabilizado apenas os quantitativos, desconsiderando o agrupamento de cenários ou combinações, e, ora estudou-se as indicações de erros sob o aspecto de grupos de obras com as mesma características quanto a(s) fase(s) que originaram os erros construtivos.

Além disso, o Gráfico 3 e a Tabela 3 ilustram ainda que, de modo geral, o ranking número 1 no que tange as não conformidades em obras de engenharia civil contempladas na amostragem de 113 documentos, para análise nesta pesquisa, encontram-se combinadas em projeto e execução. Em outras palavras, observou-se que as inadequações nas fases de projeto e execução simultaneamente, foram preponderantes para o surgimento de erros na construção civil.

Da mesma forma, o segundo lugar corresponde às obras que apresentaram erros e inadequações nas etapas de execução e manutenção ao mesmo tempo, com 21 apontamentos que correspondem a 18,6\% dos casos. E, seguindo em terceira posição, tem-se um quadro crítico onde deparou-se com falhas em todas as etapas da obra, isto é, nas fases de projeto, execução e manutenção concomitantemente.

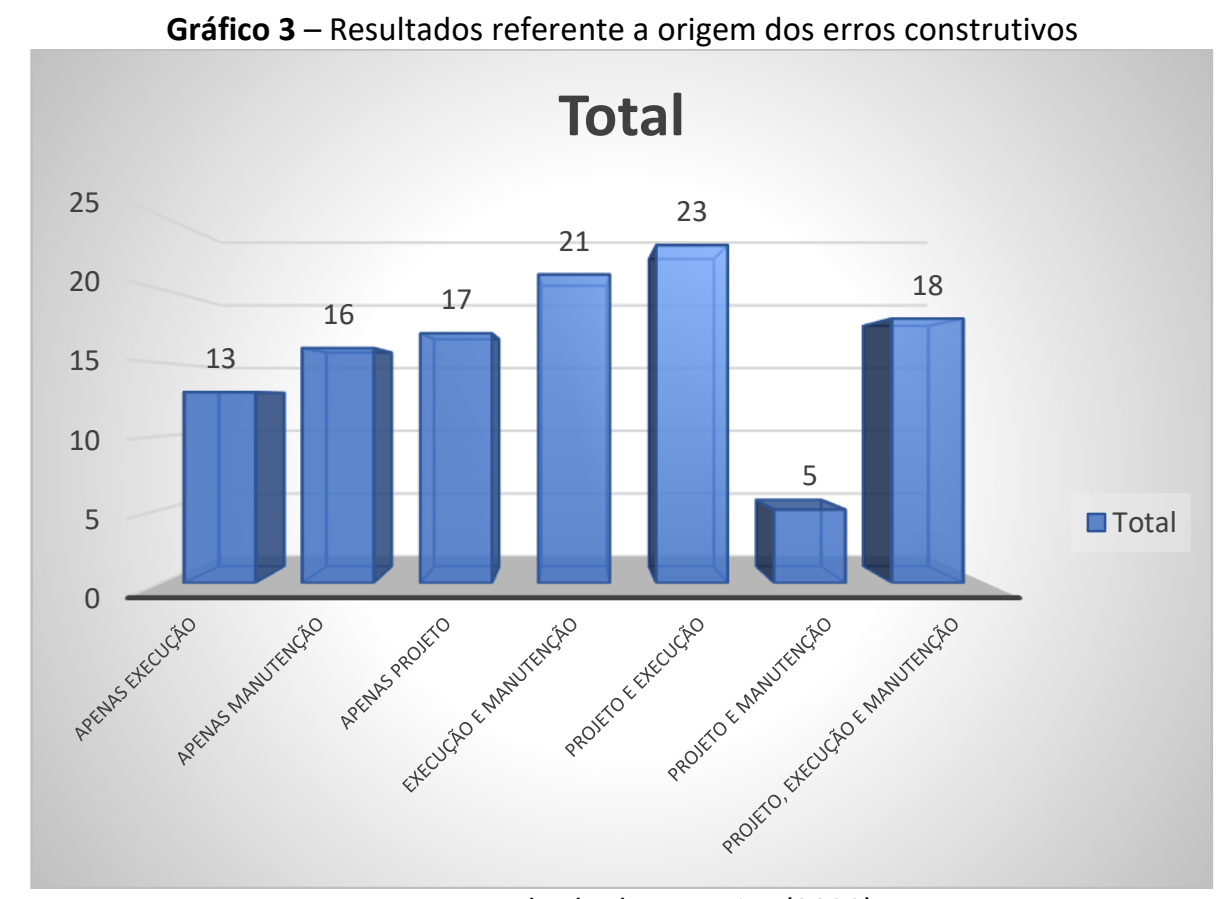

Fonte: Resultado da pesquisa (2020).

Tabela 3 - Resultados referente a origem dos erros construtivos

\begin{tabular}{l|cc|}
\hline Rótulos de Linha & Contagem de Status Geral & Percentual (\%) \\
\hline Apenas Execução & 13 & $11,5 \%$ \\
Apenas Manutenção & 16 & $14,2 \%$ \\
Apenas Projeto & 17 & $15,0 \%$ \\
Execução e Manutenção & 21 & $18,6 \%$ \\
Projeto e Execução & 23 & $\mathbf{2 0 , 4 \%}$ \\
Projeto e Manutenção & 5 & $4,4 \%$ \\
Projeto, Execução e Manutenção & 18 & $15,9 \%$ \\
\hline Total Geral & $\mathbf{1 1 3}$ & $\mathbf{1 0 0 , 0 \%}$ \\
\hline
\end{tabular}

Fonte: Resultado da pesquisa (2020).

Logo, a partir desse levantamento e apuração de dados, notou-se que as manifestações de erros e não conformidades construtivas estão presentes em mais de uma etapa, por corresponderem a 59,3\% dos casos desta análise, como pode ser observado na Tabela 4, ou seja, é possível perceber que se erram em mais fases dentro do processo construtivo, podendo impactar drasticamente nas propostas de soluções e correções no que tange a reparação de danos, encarecendo-as e até mesmo inviabilizando-as. 
Tabela 4 - Resultados referentes a origem dos erros construtivos - Por cenário

\begin{tabular}{|c|c|c|c|c|}
\hline Rótulos de Linha & $\begin{array}{l}\text { Contagem de } \\
\text { Status Geral }\end{array}$ & $\begin{array}{l}\text { Percentual por } \\
\text { Status(\%) }\end{array}$ & Cenário & $\begin{array}{c}\text { Percentual por } \\
\text { Grupo (\%) }\end{array}$ \\
\hline Apenas Execução & 13 & $11,5 \%$ & \multirow{3}{*}{$\begin{array}{c}\text { Ocorrência em } \\
\text { apenas uma } \\
\text { etapa }\end{array}$} & \multirow{3}{*}{$40,7 \%$} \\
\hline Apenas Manutenção & 16 & $14,2 \%$ & & \\
\hline Apenas Projeto & 17 & $15,0 \%$ & & \\
\hline Execução e Manutenção & 21 & $18,6 \%$ & \multirow{4}{*}{$\begin{array}{c}\text { Ocorrência em } \\
\text { mais de uma } \\
\text { etapa }\end{array}$} & \multirow{4}{*}{$59,3 \%$} \\
\hline Projeto e Execução & 23 & $20,4 \%$ & & \\
\hline Projeto e Manutenção & 5 & $4,4 \%$ & & \\
\hline Projeto, Execução e Manutenção & 18 & $15,9 \%$ & & \\
\hline Total Geral & 113 & $100,0 \%$ & - & $100,0 \%$ \\
\hline
\end{tabular}

Fonte: Resultado da pesquisa (2020).

\section{Conclusões}

A engenharia civil está além de projetar e construir obras, visto que, ela é a grande responsável por proporcionar o desenvolvimento das cidades e por mostrar-se indispensável para a ampliação da infraestrutura, bem como, para a resolução de problemas de caráter econômico e social.

Dessa forma, nota-se que a indústria civil é extremamente relevante desde sua origem até os tempos atuais, especialmente por se tratar de um dos grandes motores econômicos e de desenvolvimento continuo de um país, capaz de promover obras destinadas à moradia, comércio, lazer, urbanização etc., promovendo o bem estar da sociedade, oferecendo melhores condições para o ser humano.

Nesse cenário, sabe-se que as atividades relacionadas ao mercado da construção civil exigem de todos os agentes nesse processo uma adequação estratégica e operacional frente ao processo construtivo desde à qualidade, desempenho e ausência de não conformidades, uma vez que, quando não são submetidas à análises e inspeções podem comprometer a funcionalidade e segurança da estrutura, podendo provocar colapsos atingindo diretamente a segurança de pessoas e outros bens, convocando, para tanto, ações e cuidados a serem adotados durante todas as fases da construção em prol de eliminar falhas.

No entanto, percebe-se que a ocorrência de erros e inconformidades construtivas está cada vez mais presente e recorrente em obras de engenharia civil pelo mundo, originando em todas as fases do processo de construção, havendo inclusive acúmulo de erros em mais de uma etapa, independente da sua finalidade.

Para tanto, a partir deste estudo foi possível notar que as manifestações patológicas são sintomas de erros originados na fase de projeto, execução e manutenção, e, sobretudo, com relação a esta pesquisa na qual avaliou-se 113 publicações acerca do tema, concluiu-se que a fase crítica do processo construtivo em que deparam-se o maior número de ocorrências de falhas e não conformidades encontra-se primeiramente na etapa de execução da obra, seguido da fase projetual, contudo, ainda que ocupando a última colocação, percebeu-se que o período compreendido pela manutenção também apresentou números de eventos representativos e significativos e que não devem ser desprezados na amostra.

Ressalta-se ainda que, o mercado da construção civil submete-se, constantemente, a inovações e adequações, mediante o avanço tecnológico que promove a inserção de novos produtos, ferramentas, sistemas, entre outros insumos. Assim, o cenário atual, deve ser observado e estudado com frequência, para que se possa buscar soluções e intervenções capazes de eliminar ou ao menos reduzir tais ocorrências, e, além disso, trazer resultados atualizados para o cenário da construção civil.

Ademais, conclui-se por meio da apuração dos resultados e dados levantados nesta pesquisa que, as manifestações de erros e não conformidades construtivas, presentes em mais de uma etapa, representam cerca de 59\% dos acontecimentos desta análise. Isto é, erram-se em diversos momentos do processo, o que potencializa no encarecimento de técnicas e soluções corretivas, podendo inclusive inviabilizá-las em determinadas situações, ou até mesmo provocar danos irreparáveis, quando comprometem a segurança dos usuários. 


\section{Referências Bibliográficas}

Bomtempo, Tullio Barros Silva. Engenharia civil forense: Principais causas de incidentes em obras de engenharia civil e procedimentos de investigação. Acta de Ciências e Saúde, v. 2, n. 1, p. 1-13, 2017.

CBIC, Câmara Brasileira da Indústria da Construção. Desempenho de Edificações Habitacionais: Guia Orientativo para Atendimento à Norma ABNT NBR 15575/2013. 2 ed. Brasília: Gadioli Cipolla Comunicação, 2013.

Correia, Flaviana Silva Moraes et al. Análise dos principais problemas construtivos decorrentes de falhas de projeto - Estudo de caso em Maceio-AL. Caderno de Graduação-Ciências Exatas e Tecnológicas-UNITALAGOAS, v. 4, n. 2, p. 57, 2018.

Cremonini, Ruy Alberto. Incidência de Manifestações Patológicas em Unidades Escolares da Região de Porto Alegre: Recomendações para projeto, execução e manutenção. Dissertação de Mestrado em Engenharia Civil. Escola de Engenharia da Universidade Federal do Rio Grande do Sul, Porto Alegre, 1988. Disponível em: <http://www.lume. ufrgs.br/>. Acesso em: 12 out. 2019.

Fabrício, Marcio Minto. Projeto Simultâneo na construção de edifícios. São Paulo, 2002. Tese de Doutorado em Engenharia de Construção Civil e Urbana. Escola Politécnica, Universidade de São Paulo.

Helene, Paulo R. L. Manual para Reparo, Reforço e Proteção de Estruturas de Concreto. 2 ed. São Paulo: Red Rehabilitar, 2003.

Iantas, Lauren Cristina. Estudo de caso: Análise de Patologias Estruturais em Edificação de Gestão Pública. Dissertação de Monografia, Especialização em Construção de Obras Públicas. Universidade Federal do Paraná, Curitiba, $2010 . \quad$ Disponível em: <https://acervodigital.ufpr.br/bitstream/handle/1884/34354/IANTAS,\%20LAUREN\%20CRISTINA.pdf>. Acesso em: 12 out. 2019.

Isoppo, João Octávio Justo; BERNARDINO, Leonel Brignoli. Erros de projeto na execução de obras: estudo de caso em uma escola de educação básica no município de Sombrio-SC. Tubarão, 2017.

Kato, Ricardo Bentes. Comparação entre o sistema construtivo convencional e o sistema construtivo em alvenaria estrutural segundo a teoria da construção enxuta. 2002.

Marcelli, Maurício. Sinistros na Construção civil: causas e soluções para danos e prejuízos em obras. São Paulo: Pini. 2007.

Oliveira, Daniel Ferreira. Levantamento de Causas de Patologias na Construção Civil. Trabalho de Conclusão de Curso em Engenharia Civil. Universidade Federal do Rio de Janeiro, 2013. Disponível em: <http://monografias.poli.ufrj.br/monografias/monopoli10007893.pdf>. Acesso em: 12 out. 2019.

Romano, Fabiane V. Modelo de Referência Para o Gerenciamento do Processo de Projeto Integrado de Edificações. Florianópolis, 2003. $381 \mathrm{f}$. Tese de Doutorado em Engenharia Civil. Escola de Engenharia, Universidade Federal de Santa Catarina, Florianópolis, 2003.

Trindade, Diego dos Santos. Patologia em Estruturas de Concreto Armado. Trabalho de Conclusão de Curso em Engenharia Civil. Universidade Federal de Santa Maria, 2015. Disponível em: <http://coral.ufsm.br/engcivil/images/PDF/2_2015/TCC_DIEGO\%20DOS\%20SANTOS\%20DA\%20TRINDA DE.pdf>. Acesso em: 12 out. 2019.

Tzortzopoulos, Patrícia. Contribuições para o desenvolvimento de um modelo do processo de projeto de edificações em empresas construtoras incorporadoras de pequeno porte. Dissertação de mestrado em engenharia civil, Universidade Federal do Rio Grande do Sul. Porto Alegre, 1999.

Vanni, Claudia Maria Kattah; Gomes, Abdias Magalhães; Andrey, Paulo Roberto Pereira. Análise de falhas aplicada à otimização de projetos de edificações. XIX ENCONTRO NACIONAL DE ENGENHARIA DE PRODUÇÃO, Rio de Janeiro, 1999.

Vieira, Rubia Nunes. Investigação Sobre a Origem de Erros e Inconformidades em Obras de Engenharia Civi. Trabalho de conclusão de curso em Engenharia Civil. Pontificia Universidade Católica de Minas Gerais. Minas Gerais, Belo Horizonte, 2020. Disponível em: <http://bib.pucminas.br:8080/pergamumweb/vinculos/00006d/00006dc3.pdf>. 